\title{
Efficiency enhancement using optimized static scheduling technique in TSCH networks
}

\author{
Manjunath G. Asuti, Prabhugoud I. Basarkod \\ School of Electronics and Communication Engineering, REVA University, India
}

\begin{tabular}{|c|c|}
\hline Article Info & ABSTRACT \\
\hline Article history: & In recent times, the reliable and real-time data transmission becomes \\
\hline Received Jun 5, 2019 & $\begin{array}{l}\text { a mandatory requirement for various industries and organizations due to } \\
\text { the large utilization of Internet of Things (IoT) devices. However, the IoT }\end{array}$ \\
\hline Revised Oct 28, 2019 & devices need high reliability, precise data exchange and low power utilization \\
\hline Accepted Nov 6, 2019 & $\begin{array}{l}\text { which cannot be achieved by the conventional Medium Access Control } \\
\text { (MAC) protocols due to link failures and high interferences in the network. }\end{array}$ \\
\hline Keywords: & $\begin{array}{l}\text { Therefore, the Time-Slotted Channel Hopping (TSCH) networks can be used } \\
\text { for link scheduling under the IEEE } 802.15 .4 \mathrm{e} \text { standard. In this paper, }\end{array}$ \\
\hline IEEE $802.15 .4 \mathrm{e}$ & we propose an Optimized Static Scheduling Technique (OSST) for the link \\
\hline & $\begin{array}{l}\text { scheduling in IEEE } 802.15 .4 \mathrm{e} \text { based } \mathrm{TSCH} \text { networks. In USST the link } \\
\text { schedule is optimized by considering the packet latency information during }\end{array}$ \\
\hline MAC protocol & transmission by checking the status of the transmitted packets as well as \\
\hline Time-slotted channel hopping & keeping track of the lost data packets from source to destination nodes. \\
\hline Wireless sensor networks & $\begin{array}{l}\text { We evaluate the proposed OSST model using 6TiSCH Simulator and } \\
\text { compare the different performance metrics with Simple distributed TSCH } \\
\text { Scheduling. }\end{array}$ \\
\hline
\end{tabular}

Copyright $(0) 2020$ Institute of Advanced Engineering and Science. All rights reserved.

\section{Corresponding Author:}

Manjunath G.Asuti,

School of Electronics and Communication Engineering,

REVA University,

Rukmini Knowledge Park, Kattigenahalli, Yelahanka, Bengaluru-560064, India.

Email: manjunathasuti82@gmail.com

\section{INTRODUCTION}

In recent times, the significance of Internet of Things (IoT) scenarios has enhanced drastically and has become one of the booming technologies which can be utilized to exchange data between objects and humans. Also, it helps to monitor the enormous amount of data in real time scenarios in various industries, several universities, organizations and healthcare applications etc. However, the integration of sensor nodes is a complex and expensive process which can be sorted out by utilizing Wireless Sensor Network (WSN) standards like IEEE 802.15.4e and Wireless Hart etc. The IEEE 802.15.4e $[1,2]$ standard is used in many Internet of Things (IoT) networks which employs the Time-Slotted Channel Hopping (TSCH) mode in the Medium Access Control (MAC) layer. TSCH has become a promising and very interesting topic among the various researchers all over the world due to its high reliability and packet transmission capacity. TSCH mode works under MAC protocol to ensure high reliability and low power by multiplexing in both time and frequency domain. TSCH can handle frequency of nodes in an efficient manner which is highly crucial for IoT scenarios.

Moreover, in existing TDMA enabled techniques, interference occurrence becomes an unsolved issue due to the multi-path fading and external interferences between adjacent networks. This issue can be handled efficiently using channel hopping which can transmit different packets at several channels in a synchronized manner. Therefore, a combination of TDMA and Channel Hopping can be utilized to enhance efficiency, reliability and stability [3]. 
However, there are some issues like node mobility, high energy consumption and high overhead which need to be addressed soon in order to maintain the performance of the TSCH networks. The mobility reduction of nodes can further led to network dis-connectivity which can initiate the functionality disruptions. Also due to high overhead, the energy consumption in the network can be drastically enhanced [4]. Therefore, in order to maintain high energy efficiency and enhance network connectivity MAC protocols must have speedy and smooth mechanism under IEEE 802.15.4e standards. The sensor nodes remain in synchronization [5] using TSCH technique and time slots are introduced in the slot frames. The sensor nodes are utilized to perform operations like transmission, reception and sleep in a scheduled way. The channel hopping ability of TSCH mode helps to avoid issues like multipath fading and environmental interference. TSCH mode enhances robustness of the network and decreases the effect of collision and enhance throughput of the network by prolonging communication range. The IEEE 802.15.4e standard operating in TSCH mode provides the basic scheduling [6] of the nodes in a network, but it does not provide schedules which can improve the low data rates, reliable communication and energy efficiency in the current industrial applications. Thus, in recent years, several researchers have presented different techniques which can increase the performance of TSCH networks and to reduce the effect of high energy consumption and overhead.

In this paper, we propose an Optimized Static Scheduling Technique (OSST) for scheduling of the nodes in a TSCH network to provide several timeslots for sender and receiver nodes based on their slot level utilization. OSST provides an effective and optimal solution in case of high traffic load when compared to any other existing techniques like simple static scheduling. This paper is organized in following sections, which are as follows. In section 2 , we present related work in the field of scheduling in TSCH networks. In section 3, we describe the proposed Optimized Static Scheduling Technique (OSST) model. In section 4, we present the experimental results and performance evaluation, and section 5 concludes our paper.

\section{RELATED WORK}

In [7], proposed an Autonomous TSCH scheduling mechanism for dynamic networks in which the nodes compute their schedules without any requirement of central or distributed scheduler. In [8], a TSCH technique is introduced based on Multiple Access Control (MAC) protocol to enhance the reliability and connectivity of the network. Here, the scheduling of slots is completed in a distributed way with local time synchronization. However, high delay occurs using this technique. In [9], a TSCH technique is adopted for IoT devices to improve the management of Information Centric Networking (ICN) packets. This technique will reduce the high congestion in the network and provide better connectivity. In [10], the k-cast scheduling is proposed to increase the reliability of a TSCH network by adapting a single transmitter and $\mathrm{k}$ receivers.

In [11], A Novel Link-based scheduling is proposed to allocate a cell for a pair of nodes in a network. In [12], the scheduling mechanism is based upon synchronization of all the nodes in a TSCH networks by incorporating a trickle timer for beacon scheduling. In [13], a novel enhanced beacon scheduling is proposed in IEEE 802.15.4e-TSCH networks which reduce the power consumption and collisions. In [14], a TSCH technique is adopted which rely upon Medium Access Control (MAC) protocols to provide better end-to-end connectivity and high reliability. This technique helps to reduce interference in multi-hop networks by balancing between the reliability and packet data delivery.

In [15], a distributed scheduling algorithm is proposed for traffic flow in all the directions in a IEEE 802.15.4e- TSCH networks. In [16], a new scheduling algorithm is proposed for resolving the conflicts in a TSCH networks by adapting a matching rule by reducing energy consumption and delay. In [17], a Poisson prediction modeling is used for scheduling in TSCH networks which reduces the end-to-end latency, overhead and queue length. In [18], a TSCH enabled scheduling technique is introduced for IoT enabled devices. Here, a QSS algorithm is adapted which reduces the quantity of information packets needed for scheduling. In [19], a TSCH scheduling technique is designed for IoT devices in an Information-Centric Networking environment which helps to avoid the error recovery issues in the ICN environment. However, it requires more delay for scheduling. In [20], Adaptive medium selection technique is adopted to reduce the delay in TSCH networks. It ensures high reliability and efficiency while delivering the data packets. In [21], an efficient scheduling technique is adopted in TSCH networks to provide high throughput and minimum delay considering the deadline constraints. A scheduler based on genetic algorithm is proposed to provide optimal throughput and minimum complexity. However, optimization problem occurs using this technique.

In [22], focused on energy constraint in a IEEE 802.15.4e-TSCH Network to schedule the nodes by incorporating a heuristic scheduling method. In [23], an efficient scheduling technique is introduced in TSCH networks under IEEE 802.15.4 protocols to handle high traffic. It is used to estimate the high rate of 
congestion to its maximum capability by enhancing the reliability and efficiency of the TSCH networks. However, the computational complexity is high using this scheduling. In [24], a centralized scheduling technique is introduced to ensure high reliability and performance in TSCH networks. This technique enhances the throughput of the network and reduces the delay problems. However, maximum weight matching and delay minimization problems may decrease the performance of the network.

In [25], the performance analysis of Orchestra is investigated for TSCH Networks used in Internet of Things (IoT) applications. In [26], a new scheme is introduced for scheduling TSCH networks by allocating Virtual slots for the nodes in a TSCH networks which doesn't require any additional reconfiguration. In [27], an autonomous scheduling mechanism is introduced to minimize the delay occurred due to packet transmission in a TSCH networks. In [28], an analytical model is proposed for TSCH-CSMA for the shared links in a TSCH networks.

\section{PROPOSED OPTIMIZED STATIC SCHEDULING TECHNIQUE (OSST)}

\subsection{Preliminaries}

In this section, the detailed modeling of Time-Slotted Channel Hopping (TSCH) technique is introduced. Let $\mathrm{N}$ represent the number of channels in a TSCH networks for communication. The range of this channel lies from $[0, \mathrm{~N}]$ and every channel can be termed as a channel equalizer. For the offered time-slot and channel equalizer, various connections of pair wise type is provided to communicate with different devices. Assume that [m, channel equalizer] shows a connection between a node pair. Then, for a $\mathrm{m}$ timeslots with a network frequency $f$ specific time duration can be described as,

$$
f=G\left[(S+R) \% M_{C}\right]
$$

Where, $S$ can be termed as non-varied slot number and can be described as the overall time-slots exhausted from the network initiated point. Global increment method is utilized for non-varied slot number $S$ updation at each time-slot. Therefore, it is utilized for nodes and can be termed as timeslot counter. Here, $R$ can be termed as channel equalizer whereas $C$ denotes the channels utilized in the network. Here, $G$ can be described as a lookup table function. Furthermore, equation (1) shows a channel hopping method which provides varied frequency for a varied time-slot in a single link. This verifies that any available channel can be utilized from the network link to communicate between devices. Basically, TSCH scheduling technique offers two dedicated links namely single and shared links. Shared links possess a vital role in TSCH scheduling due to their routing and scheduling data transmission to other destination, also to reduce the repetitive collision probability.

\subsection{Modeling of time-slotted channel hopping with collision avoidance (TSCH-CA)}

This section provides modeling of TSCH-CA Algorithm which decreases the repetitive collision probability in case of a shared links in TSCH networks. A sender node $t$ transmits an information packet to the receiving node $n$ over a shared link $(t, n)$. The sender node $t$ transmits the consecutive information packets, once the first information packet is reached to the destination. Thus, collision avoidance algorithm is introduced to decrease the repetitive collisions. The following steps need to be followed to have an efficient TSCH-CA [28] technique.

a. A state variables group need to be initialized like total iterations required for proper re-transmissions for the current frame $(I=0)$ and exponent $E$.

b. An arbitrary constant $v \in\left[0,2^{E}-1\right]$ is introduced.

c. The data packets which need to be re-transmitted on $v$ shared channels can be delayed till the dedicated link is experienced.

d. If the data packets are re-transmitted on $v$ shared channels successfully then $E$ can be set as $J_{d \downarrow}(E)$ and the process can be finished. In case, the data packets transmission becomes unsuccessful then the state parameters can be updated as follows, $I=I+1$ and $E=\min \left(E+1, J_{d \uparrow}(E)\right)$. At last, if the total retransmissions become larger than the threshold value $\left(I>J_{d_{\uparrow}}(E)\right)$ for the present frame then the data packets can be released or the second step can be repeated again.

Here, the energy required for the transmission and reception of data packets can be denoted as $F_{t}$. Then, the power required for the transmission to reception of data packets can be expressed as $Q_{k y}\left(Q_{n y}\right)$. Here, data acknowledged sensing time is denoted as $B_{k y}\left(B_{a}\right)$. Therefore,

$$
F_{Y_{k, y} \cdot Y_{k, x}+1}=m \cdot F_{t}
$$


Where $F_{Y_{k, y} . Y_{k, x}+1}$ represents the stored energy from state $Y_{k x}$ to the consecutive state $Y_{k x}+1$. Here, $F_{t}$ can be described as,

$$
F_{t}=Q_{k y} \cdot B_{k y}+Q_{n y} \cdot B_{a}
$$

Then, the estimated probability $H(e)$ of weak connections $e \in A_{i}\left(D_{i}\right)$ can be expressed as where $A_{i}\left(D_{i}\right)$ is the group of total weak connections in the network of nodes $D_{i}$,

$$
H(e)=\left(\begin{array}{c}
D_{i} \\
e_{L_{\mathbb{1}+1}} e_{P_{\mathfrak{I}+11}} \cdots \cdots e_{P_{\mathfrak{I}+1 v_{\mathfrak{I}+1}-1}}
\end{array}\right) \cdot\left(\left(V_{\mathbb{I}+1}\right)^{-1}\right)^{D_{\mathbb{I}}}
$$

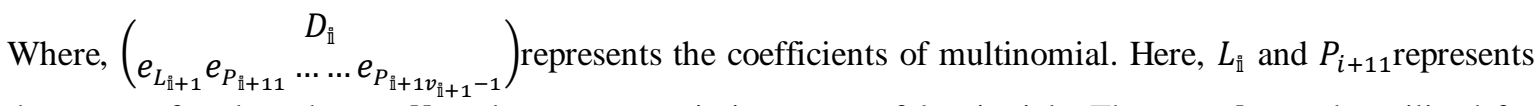
the states of nodes whereas $V_{i+1}$ denotes transmission states of $i+1$ trials. The state $L_{i}$ can be utilized for next transmission. In equation (4), the second term can be expressed as the probability of occurrence of every trial. Then, stochastic network transition $A_{\mathfrak{j}}$ can be expressed as $A_{\mathfrak{j}}=\left\{e_{0}, e_{1}, \ldots, e_{\uparrow N-1}\right\}$. Whenever, failure occurs in nodes $D_{0}$ transmission in state $L_{0}$, similarly failure occurs in nodes $D_{1}$ transmission in state $L_{1}$ and so on, then the stochastic set $\varphi_{A_{i}}$ for each stochastic network transition $A_{j}$ can be expressed as,

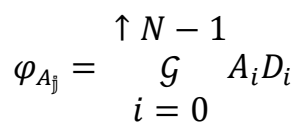

Then, the stochastic network transition $A_{j} \in \varphi_{A_{j}}$ probability $Q\left(A_{j}\right)$ can be expressed as,

$$
Q\left(A_{j}\right)=\prod_{i=1}^{\uparrow N-1} H\left(e_{i}\right)
$$

Where, $Q\left(A_{j}\right)$ can be obtained using (4). The occurrence of stochastic network transition $A_{j} \in \varphi_{A_{j}}$ can progress in next stage. Moreover, the energy $F_{Y_{k, y} . Y_{k, x}+1}$ required for the transmission from one state to next state can be expressed in the following equation,

$$
F_{Y_{k, y} \cdot Y_{k, x}+1}=m \cdot F_{e}
$$

Where, transmission energy $F_{e}$ can be expressed as,

$$
F_{e}=Q_{k y} \cdot B_{k y}+Q_{n y} \cdot B_{k o}
$$

Where, $B_{k o}$ can be described as the connection timeout. Then, the energy $F_{Y_{k, y}, Y_{k, x}+1}$ required for the transmission from one state to next state, when only one node successfully transmit its packets and the other $(m-1)$ nodes experience failure in packet transmission,

$$
F_{Y_{k, y} Y_{k, x}+1}=F_{t}+(m-1) \cdot F_{e}
$$

Assume that, compression energy evaluation occurs with estimated probability $Q_{C E}(m)$. In all $m$ receiving nodes, the node $l$ send its data packets successfully and changes to state $T$ and every remaining node $m-1$ failed to transmit due to failure and need to resend again. Our proposed model has total $N+1$ conditions which belongs to each possible state of node $l$ i.e. $L_{0}, L_{1}, \ldots \ldots, L_{\uparrow N}$. Assume that, the transmitted node $l$ lies in the state $L_{y}(0 \leq y \leq \max \uparrow)$. Then, the probability of node $l$ in state $L_{y}$ at time $k_{x}$ can be determine as when $\sum_{y=0}^{\uparrow N} Q_{\mathbb{C E}}^{y}=Q_{C E}(m)$,

$$
Q_{C E}^{y}=Q_{C E}(m) \cdot\left(Y_{k_{x}}\left[L_{y}\right] \cdot(m)^{-1}\right)
$$

Where, the node $l$ changes to state $T$, then the remaining node $Y_{k_{x}}\left[L_{y}\right]-1$ nodes remains in state $L_{y}$. Moreover, the nodes present in state $L_{z}$ where $z \neq y$ receives a sending failure. Then, $f_{y}=Y_{k_{x}}\left[L_{y}\right]-1$, where $f_{z}=Y_{k_{x}}\left[L_{z}\right], z \neq y$. Moreover, the energy consumed by TSCH network can be denoted as $F_{Y_{k x} \cdot Y_{k x}+1}$, where its states changes from $Y_{k x}$ to $Y_{k x}+1$. The (11) states that only one node transmits its packets and other remaining nodes $m-1$ fails to send packets. Therefore, 


$$
F_{Y_{k x} \cdot Y_{k x}+1}=F_{t}+(m-1) \cdot F_{e}
$$

Where, probability matrix of transition, for every couple state $(Y, X)$ and $(Y, X) \in G_{t}$. Here, $Q_{Y X}$ represents the probability of network state which alters from $Y$ to $X$ state and $k_{x}$ to $k_{x+1}$ time. Similarly, $F_{Y X}$ can be defined as the energy consumed by TSCH network when the states changes from $Y$ to $X$ state. From the above equations, we define

e. Packet Transportation Probability $(\bar{B})$ : the ratio of accurately received information packets by node $n$ to the overall transmitted data packets.

f. Average information packet latency $(\bar{W})$ : The average time needed from the initial point to final point in transmitting information packets by node $n$.

g. Energy Consumption Occur $(\bar{F})$ : The total energy occurred from initial point to final point in transmitting information packets by all the nodes in TSCH network.

h. $\quad Q_{d}(k)$ : The probability of receiving at least $d$ information packets till timeslot $k$ by the node $n$.

The Packet Transportation Probability $(\bar{B})$ of state $Y_{f}^{i}$ can be determined as,

$$
\bar{B}=(M)^{-1} \sum_{i=0}^{M} i . \mu W_{\uparrow}\left[q_{i}\right]
$$

Where, the probability vector can be denoted as $\mu W_{\uparrow}=\mu_{0}\left(Q^{W_{\uparrow}}\right)$ from $W_{\uparrow}$ to $k_{0}$ timeslots. Then, the probability of correctly received information packets during time-slot $k$ can be defined as,

$$
Q(k)=\sum_{i=0}^{M-1} Q\left\{\varphi_{t_{i}}^{k}\right\} \rightarrow Q\left\{\varphi_{t_{i+1}}^{k+1}\right\}
$$

Where, the correctly packet transmission probability in the network from $i$ to $i+1$ state can be expressed as $Q\left\{\varphi_{t_{i}}^{k}\right\} \rightarrow Q\left\{\varphi_{t_{i+1}}^{k+1}\right\}$ in the time-slot $k$. Then,

$$
Q\left\{\varphi_{t_{i}}^{k}\right\} \rightarrow Q\left\{\varphi_{t_{i+1}}^{k+1}\right\}=\left\{\begin{array}{c}
Q\left\{\varphi_{t_{i+1}}^{k+1}\right\} \\
-\left[Q\left\{\varphi_{t_{i+1}}^{k}\right\}-Q\left\{\varphi_{t_{i+1}}^{k} \rightarrow\left\{\varphi_{t_{i+2}}^{k+1}\right\}\right\}\right] \\
Q\left\{\varphi_{t_{i M}}^{k+1}\right\}-Q\left\{\varphi_{t_{i M}}^{k}\right\}, \ldots \ldots \ldots \text { if } i=M-1
\end{array} \quad \text {...if } p<M-1\right.
$$

Here, the (13) can be defined as the probability of successfully packet reception at timeslot $k$ which is same as the probability of effective events in the network from slot $k$ to $k+1$. Therefore, $Q(k)$ can be defined as the summation of each probability in the time-slots $k$ to $k+1$ and the number of completed events in the network from $i$ to $i+1$. Moreover, $Q(k)$ can be termed as weights and average latency of information packets $(\bar{W})$ can be described as in time-slot $k$,

$$
\bar{W}=\sum_{k=0}^{W_{\uparrow}} k \cdot Q(k) \cdot\left(\sum_{k=0}^{\bar{W}_{\uparrow}} \mathbb{Q}(k)\right)^{-1}
$$

Where, $k \in\left[0, Q_{\uparrow}\right]$ and the average energy $(\bar{F})$ required by $M$ transmitting nodes to transmit information packets using the optimized static scheduling technique can be expressed as,

$$
\bar{F}=\delta_{Y}=\sum_{X \in G_{t}} Q_{Y X} \cdot\left(F_{Y X}+\delta_{X}\right) \forall Y \in G_{t}
$$

Where, $\bar{F}$ is the total energy required by the TSCH network to reach the state $Y_{f}^{i}$ where $0 \leq i \leq M$ and initiating from the state $Y \in G_{t}$. Then, at last, the probability of receiving at least $d$ data packets from node $n$ in initial timeslot $k$ can be denoted as $Q_{d}(k)$ and expressed as follows,

$$
Q_{d}(k)=\sum_{i=d}^{M} Q\left\{\varphi_{t_{i}}^{k}\right\}
$$

\subsection{Simple distributed TSCH scheduling (default)}

Let $\mathrm{E}$ represent a set of all schedules in a TSCH network. Let variable $\chi(t, n) \in E$ represent a data transmission schedule e and $\xi(t, n)$ represent the actual schedule at $t^{t h}$ slot on $n^{t h}$ channel. The scheduling in TSCH networks can be defined as $\sum_{\xi(t, n) \in \mathrm{E}_{0}} c(\xi(t, n))=1$, where $\mathrm{E}_{0} \subseteq E, c(\xi(t, n))$ is the probability of $\xi(t, n) \in \mathrm{E}_{0}$ and $c$ is used as heuristic in order to satisfy the condition. Data transmissions are carried out over a set of links $L_{c, n}$. Let's consider the $i^{\text {th }}$ link over which data transmission is active (state of link is active) i.e. $L_{c, n}(i)$ with a probability $Q_{i, n}$. Inactivity state of the $i^{t h} \operatorname{link}$ is $L_{c, n}(i)$ with a probability $1-Q_{i, n}$. The simple distributed (default) TSCH scheduling algorithm is described below. 


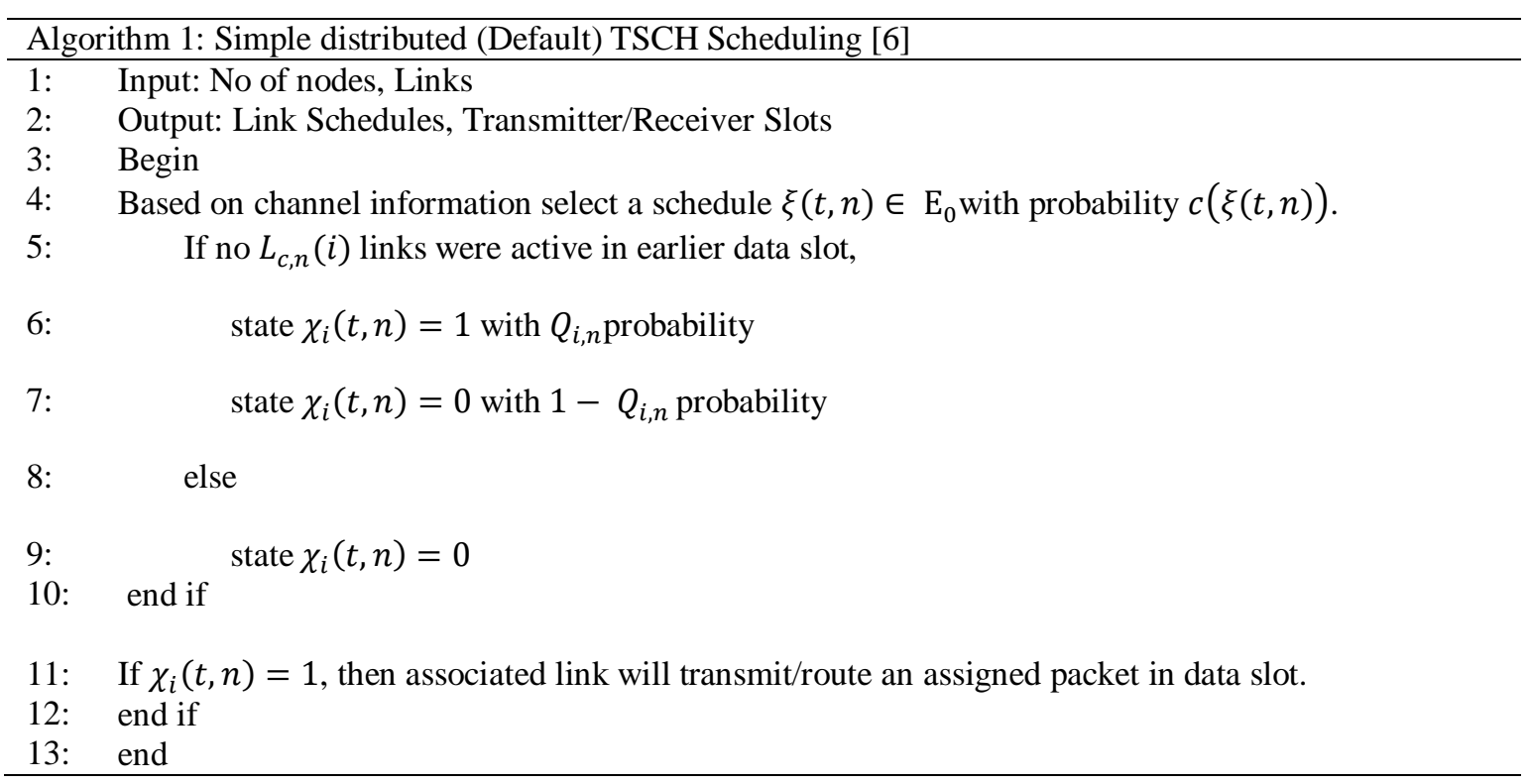

\subsection{Proposed optimized static scheduling technique (OSST)}

The Simple distributed (default) TSCH Scheduling considers only channel state information in scheduling. Proposed OSST considers packet latency information $(\bar{W})$ to control the backoff time in addition to channel state information. Packet collisions are minimized using packet latency information over links in OSST. If link latency information is included in schedule, then it can control the data-slot based carrier sensing information and optimized slot assignment, leading to improved throughput.

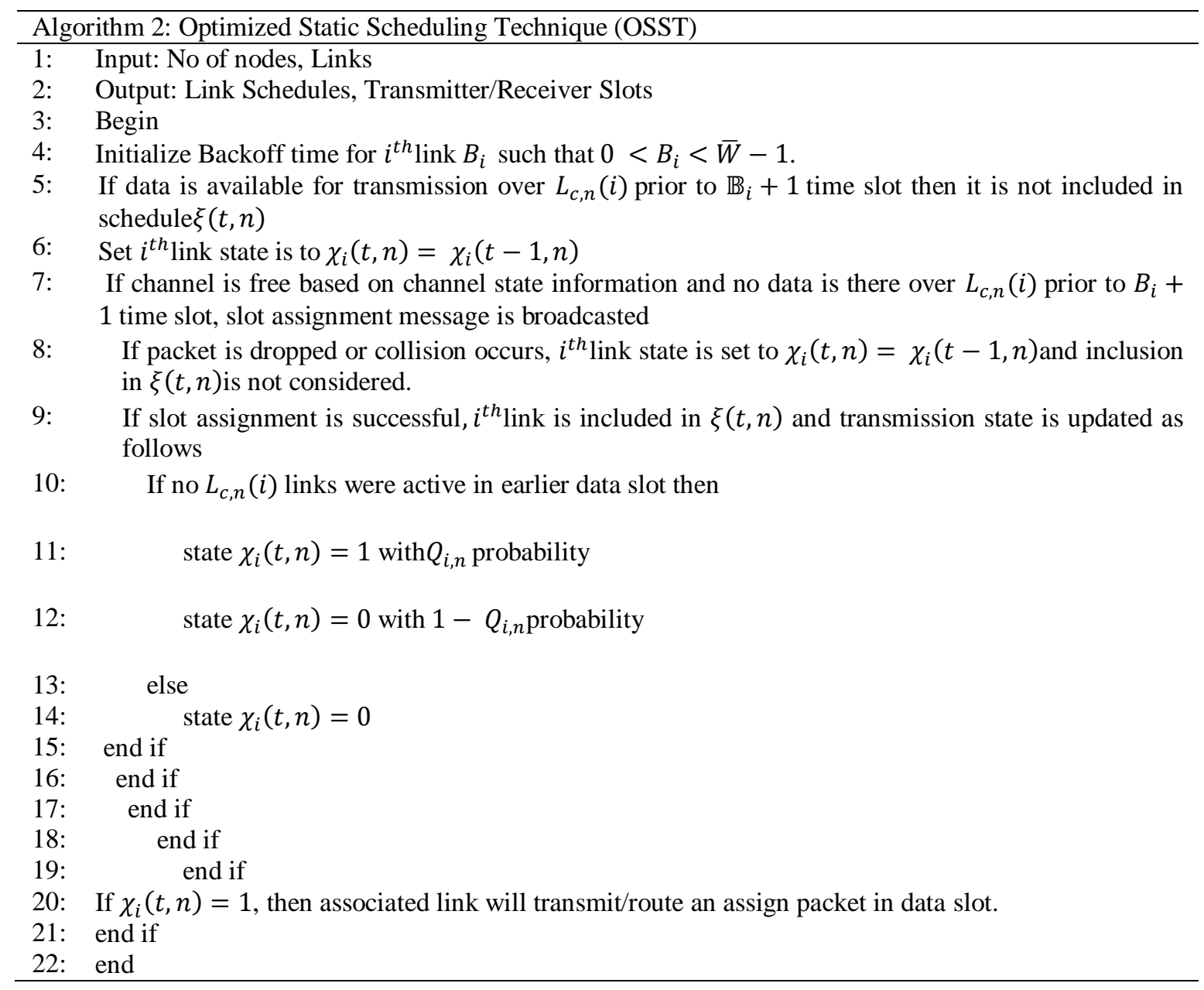




\section{PERFORMANCE EVALUATION}

This section describes about the performance of the proposed Optimized Static Scheduling Technique (OSST) for various parameters namely Efficiency, Energy Consumption, Throughput, and packet transfer rate. The proposed OSST model outperforms the default scheduling mechanism in TSCH networks. An open source 6TiSCH Simulator is used to simulate the TSCH networks with parameters listed in the Table 1 and simulated on 64-bit windows 10 OS with 16 GB RAM which contains an INTEL(R) core i5 processor.

Table 1. Simulation parameters

\begin{tabular}{ll}
\hline Parameter & value \\
\hline Frame Size & 100 slots \\
Simulation Iterations & 100 times \\
Maximum Resend packets & 8 \\
Maximum queue length & 8 \\
Total Transmitting Nodes & 4 \\
Total allocated Slots & 10 \\
Transmission data rate acknowledged & 92.6 \\
Reception data rate acknowledged & 96.3 \\
Maximum Utilization Threshold & 0.9 \\
Minimum Utilization Threshold & 0.8 \\
Traffic Rate & 5 \\
Reception Probability & 0.9 \\
\hline
\end{tabular}

Here, Figure 1 demonstrates the successful packet transmission comparison between the default scheduling and the proposed OSST model for different transmission rates in Mbps. From the Figure 1 it is clearly visible that default scheduling can perform satisfactory for lower transmission rate. However, for higher transmission rate, this technique is highly insufficient. On the other hand, the proposed OSST model performs far better for all the transmission rates. Figure 2 demonstrates the throughput of the network for the default scheduling and proposed OSST model for different transmission rates in Mbps. It is clearly visible that throughput of our proposed OSST model is much higher than the default scheduling for the different transmission rates.

Figure 3 demonstrates the number of successful packet transmission using the default scheduling and the proposed OSST model considering total number of packets transmitted. From Figure 3 it is clearly visible that successfully transmitted packets are more from total transmitted packets using proposed OSST model than default scheduling. Figure 4 demonstrates the number of packets dropped out of total number of packets transmitted using the default scheduling and proposed OSST model. Using the default scheduling technique the numbers of dropped packets are very high in comparison with total transmitted packets and number of dropped packets increases with the number of transmitted packets. However, the number of dropped packets using the proposed OSST model is minimum.

Figure 5 demonstrates the energy consumption in (mJ /reliable packets) using default scheduling and the proposed OSST model considering total number of packets transmitted. It is clearly demonstrated from Figure 5 that energy consumption is much higher using default scheduling technique than the proposed OSST model. Thus, in terms of energy consumption, proposed OSST model is much superior. Figure 6 demonstrates the energy consumption in ( $\mathrm{mJ} /$ reliable packets) using the default scheduling and proposed OSST model for the different transmission rates. The energy consumption is much higher for lower transmission rates and low for higher transmission rates using the default scheduling whereas energy consumption using the proposed OSST model is very low for all transmission rates. This verifies the superiority of the proposed OSST model in comparison with the default scheduling mechanism.

Figure 7 demonstrates the idle packets present for the default scheduling and the proposed OSST model considering total number of transmitted packets. Here, the proposed OSST model consists of large number of idle packets compared to the default scheduling mechanism. Similarly, Figure 8 demonstrates the pending packets present for default scheduling and the proposed OSST model considering the total number of transmission packets. Here, the numbers of pending packets needed to transmit are more using default scheduling when compared to the proposed OSST model. Figure 9 demonstrates the energy saved in joule using default scheduling and proposed OSST model considering the total number of packets transmitted. The proposed OSST model can save enormous amount of energy in joule, especially considering higher number of packet transmission. 


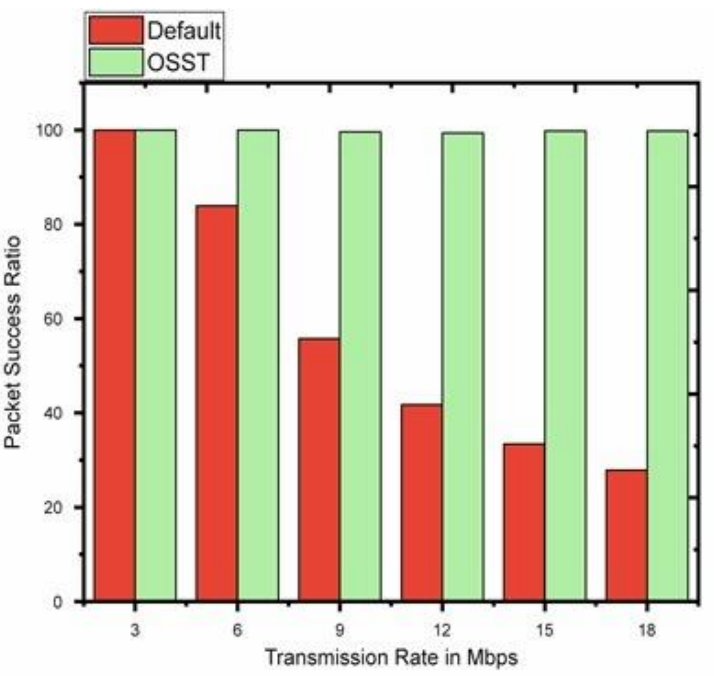

Figure 1. Packet success ratio

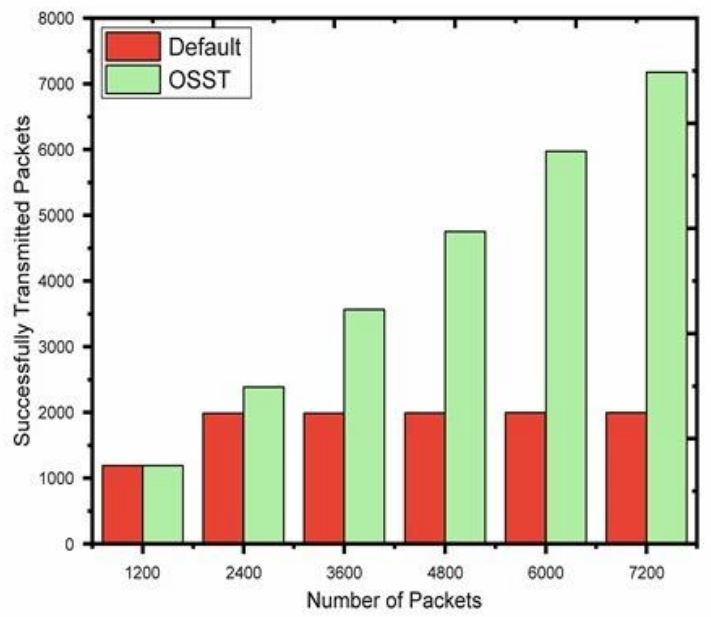

Figure 3. Successfully transmitted packets

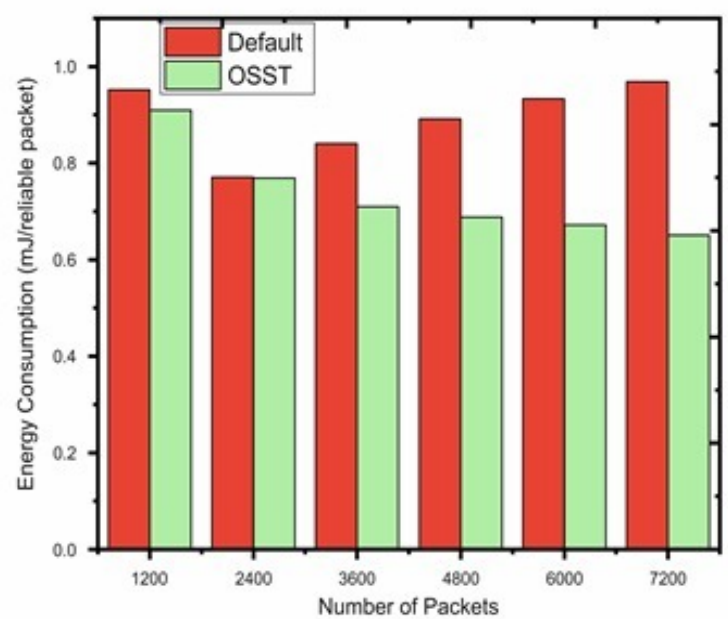

Figure 5. Energy consumption with no of packets

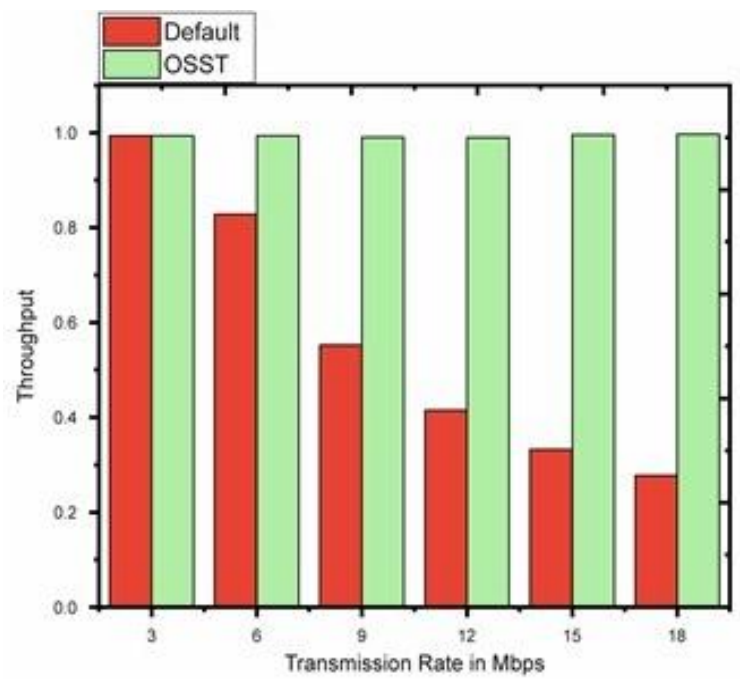

Figure 2. Throughput

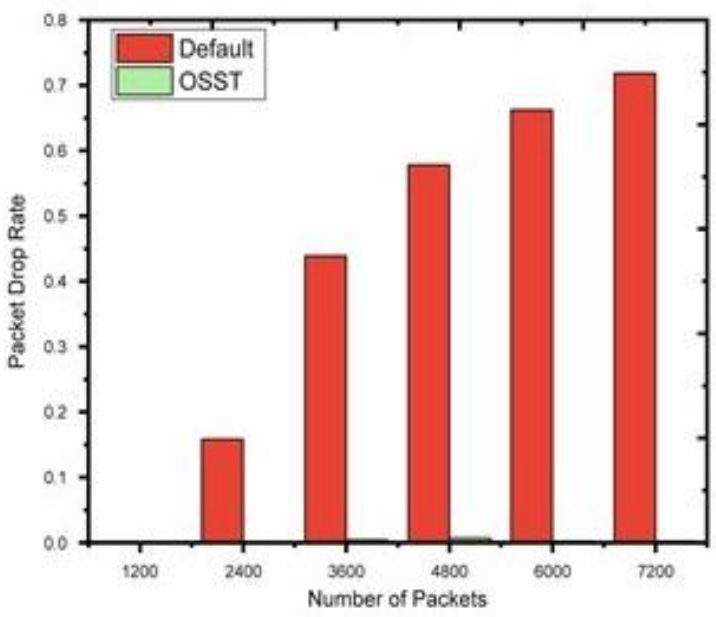

Figure 4. Packets drop rate

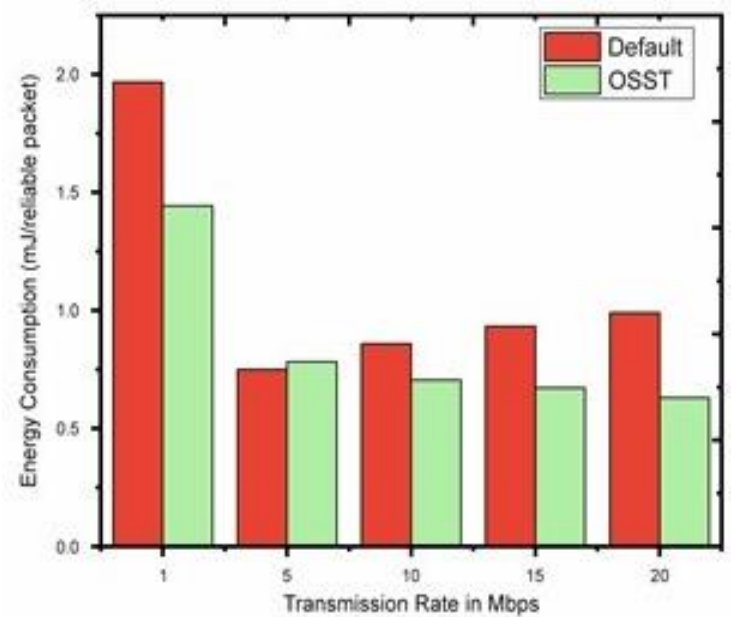

Figure 6. Energy consumption with transmission rate 


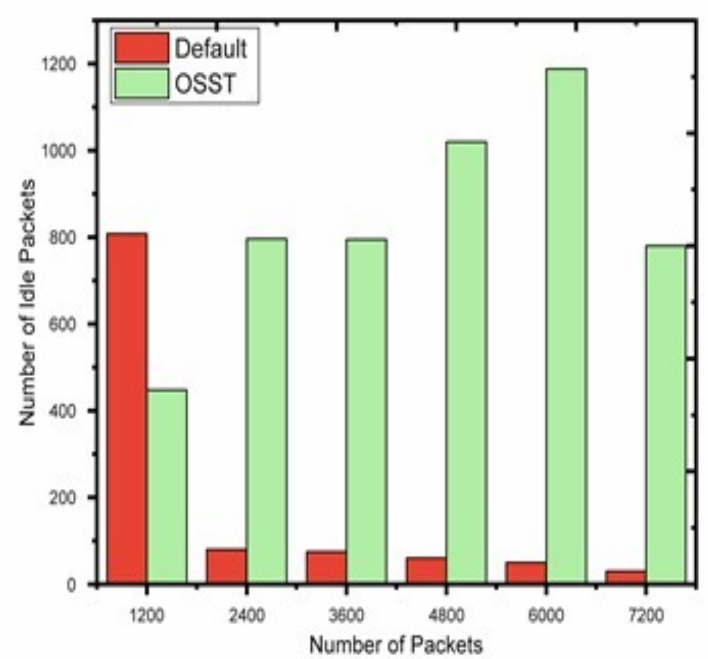

Figure 7. Number of idle packets figure

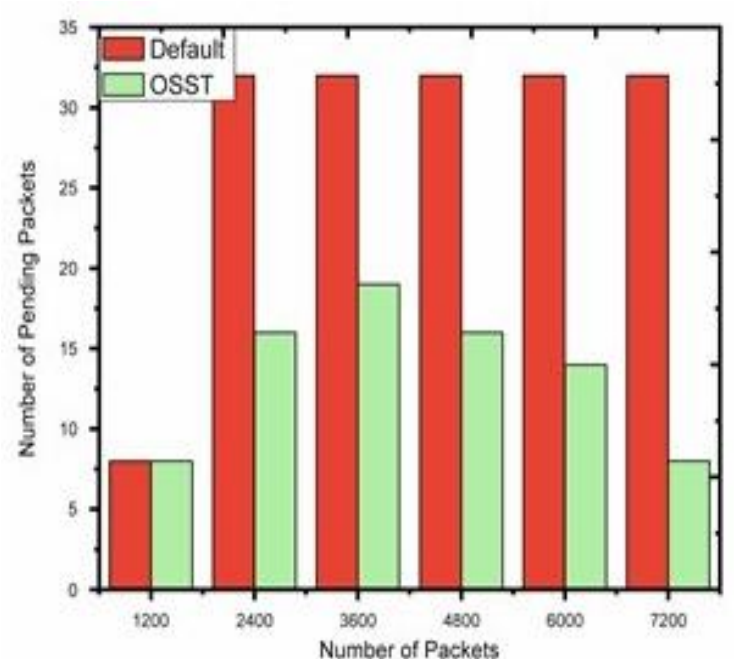

Figure 8. Number of pending packets

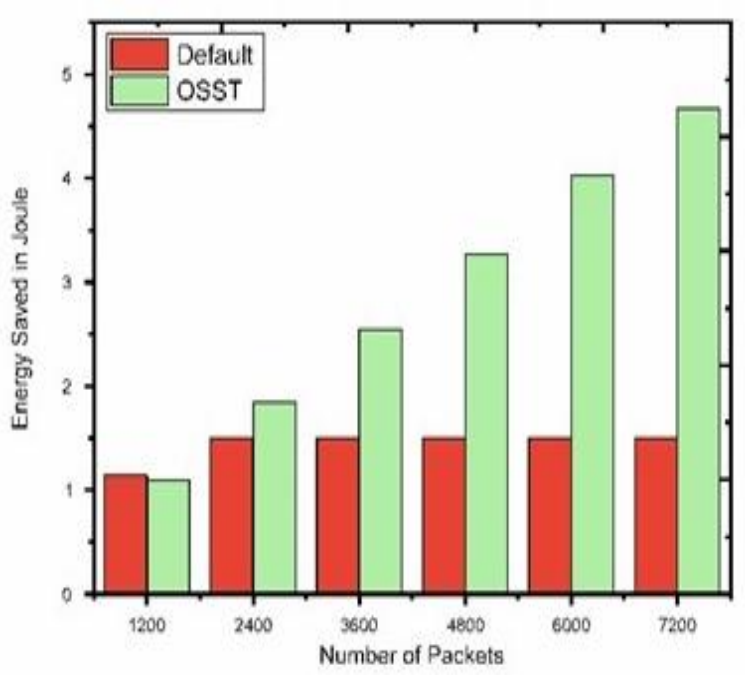

Figure 9. Energy saved in joule

\section{CONCLUSION}

IoT based developments have witnessed tremendous growth in recent times. Reliable and accurate data transmission in IoT devices can be achieved using IEEE 802.15.4e based TSCH networks. The significance of Time- Slotted Channel Hopping (TSCH) networks is extremely high under IEEE 802.15.4e standard for MAC protocols due to its high reliability, throughput and stability. To further improve the network performance, we proposed an Optimized Static Scheduling Technique (OSST) for link scheduling in IEEE 802.15.4e based TSCH networks. The proposed OSST model outperforms better when compared to the Simple distributed scheduling mechanism in terms of Energy Efficiency, Throughput, Packet success ratio, Packet drop rate for the different transmission rates.

\section{REFERENCES}

[1] Rodrigo, Antoine and Fabrice, "Scheduling for IEEE 802.15.4-TSCH and slow channel hopping MAC in low power industrial wireless networks: A Survey," Computer Communications, Elsevier, pp. 84-105, 2017.

[2] I.P.W. Group, "Part 15.4: Low-Rate wireless personal area networks (LRWPANs). Amendment 1: MAC sublayer," IEEE, IEEE Standard for Local and metropolitan area networks IEEE Std. 802.15.4e-2012, 2012.

[3] Rasool, Majid, Twan and Kees,"Dependable interference-aware time-slotted channel hopping for wireless sensor networks," ACM Transactions on Sensor Networks, vol. 14(1), 2018. 
[4] Ren-Hung, Chih-Chiang and Wu-Bin, "A distributed scheduling algorithm for IEEE 802.15.4e wireless sensor networks," Computer Standards \& Interfaces, Elsevier, pp. 63-70, 2017.

[5] Wei, Yue, Ruijie and Qin, "STS_4e: Secure time synchronization in IEEE 802.15.4e Networks," International Journal of Information Networks, Springer, 2016.

[6] K. Muraoka, T. Watteyne, N. Accettura, X. Vilajosana and K. S. J. Pister, "Simple distributed scheduling with collision detection in TSCH Networks," IEEE Sensors Journal, vol. 16(15), pp. 5848-5849, 2016.

[7] Simon, Beshr, Olaf, and Thomas, "Orchestra: Robust mesh networks through autonomously scheduled TSCH," in Proc. of the Int. Conference on Embedded Networked Sensor Systems (ACM SenSys 2015), Seoul, South Korea, 2015.

[8] H. Oh and C. T. Ngo, "A slotted sense multiple access protocol for timely and reliable data transmission in dynamic wireless sensor networks," IEEE Sensors Journal, vol. 18(5), pp. 2184-2194, 2018.

[9] Oliver, Adjih, Emmanuel, Thomas, and Matthias, "A case for time slotted channel hopping for ICN in the IoT," IEEE Journal, 2016.

[10] Ines and Fabrice, "Adaptive k-cast scheduling for high-reliability and low-latency in IEEE 802.15.4-TSCH," Springer, pp. 3-14, 2018.

[11] Seohyang, Hyung and Chongkwon, "ALICE: Autonomous link-based cell scheduling for TSCH," in proc IPSN-19, ACM, 2019

[12] Jose, David, Salvador, Javier and Victor, "A joining procedure and synchronization for TSCH-RPL wireless sensor networks," Sensors, pp. 1-20, 2018.

[13] Apostolos, Dimitrios and Christos, "Collision-Free advertisement scheduling for IEEE 802.15.4-TSCH networks," Sensors, pp. 1-22, 2019

[14] M. Vilgelm, M. Gürsu, S. Zoppi and W. Kellerer, "Time slotted channel hopping for smart metering: measurements and analysis of medium access," in proc IEEE International Conference on Smart Grid Communications (SmartGridComm), Sydney, NSW, pp. 109-115, 2016.

[15] Alper and Sedat, "DIVA: A distributed divergecast scheduling algorithm for IEEE 802.15.4e TSCH Networks," Wireless Networks, Springer, 2017.

[16] Mei, Zhu, Zhao, and Chen, "Scheduling for data transmission in Multi-Hop IEEE 802.15.4e TSCH Networks," Mobile Network Application, Springer, 2017.

[17] Taieb and Georges, "Enhanced minimal scheduling function for IEEE 802.15.4e TSCH Networks," in proc IEEE Wireless Communications and Networking Conference, Maarakesh, Morocco, 2019.

[18] Kanghoon and Sang-Hwa, "Enhanced time-slotted channel hopping scheduling with quick setup time for industrial internet of things networks," International Journal of Distributed Sensor Networks, vol. 13(6), pp. 1-14, 2017.

[19] Hahm C., Adjih E., Baccelli T., C. Schmidt and M. Wahlisch, "Designing time slotted channel hopping and information-centric networking for IoT," in proc 8th IFIP International Conference on New Technologies, Mobility and Security (NTMS), 2016.

[20] A. Elsts, X. Fafoutis, R. Piechocki and I. Craddock, "Adaptive channel selection in IEEE 802.15.4 TSCH networks," in proc Global Internet of Things Summit (GIoTS), 2017.

[21] M. Ojo, S. Giordano, G. Portaluri and D. Adami, "Throughput maximization scheduling algorithm in TSCH networks with deadline constraints," in proc IEEE Globecom Workshops (GC Wkshps), 2017.

[22] M. Ojo, S. Giordano, G. Portaluri, D. Adami and M Pagano, "An energy efficient centralized scheduling scheme in TSCH Networks," in proc IEEE Convergent Internet of Things, ICC, 2017.

[23] A. Elsts, X. Fafoutis, J. Pope, G. Oikonomou, R. Piechocki and I. Craddock, "Scheduling high-rate unpredictable traffic in IEEE 802.15.4 TSCH Networks," in proc 13th Int. Conf. on Distributed Comput. in Sensor Syst. (DCOSS), 2017.

[24] M. Ojo and S. Giordano, "An efficient centralized scheduling algorithm in IEEE 802.15.4e TSCH networks," in proc IEEE Conference on Standards for Communications and Networking (CSCN), 2016.

[25] Sana, Nouha, Mohammed, and Khalil, "A Performance analysis of ochestra Scheduling for time-slotted channel hopping networks," Internet Technology Letters, Wiley, 2017.

[26] Yuvin and Sang, "A Virtual slotframe technique for reliable multi-hop IEEE 802.15.4e time-slotted channel hopping network," International Journal of Distributed Sensor Networks, vol. 14(7), pp. 1-12, 2018.

[27] Sukho, Dong, Kim and Kangseok, "Escalator: An autonomous scheduling scheme for convergecast in TSCH," Sensors, pp 1-25, 2018.

[28] Domenico De Gulglielmo, Beshr Al Nahas, Simon Duquennoy, Thiemo Voigt and Giuseppe Anastasi, "Analysis and experimental evaluation of IEEE 802.15.4e TSCH CSMA-CA algorithm," IEEE Transactions on Vehicular Technology, vol. 66(2), pp. 1573-1588, 2017. 


\section{BIOGRAPHIES OF AUTHORS}

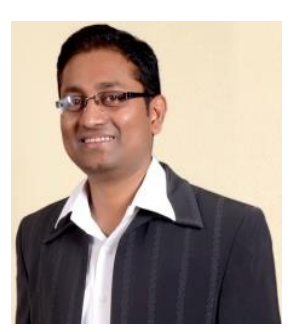

Manjunath. G. Asuti is working as Assistant Professor, School of Electronics and Communication Engineering, REVA University, Bangalore, India. He has completed B. E. in Electronics and Communication Engineering and M.Tech in VLSI and Embedded systems from Visvesvaraya Technological University, Belgaum and presently pursuing Ph.D (Wireless Sensor Networks) in REVA UNIVERSITY, Bangalore. He has 14 years of teaching experience. His areas of interest are Wireless Sensor Networks, VLSI, DSP, Embedded systems, and Communication systems. He has eight publications in reputed national/international conferences. His research interests include Digital VLSI, VHDL, FPGA, ASIC Design, DSP, Communication etc.

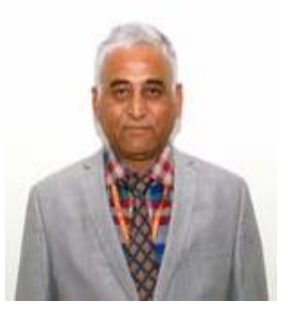

Dr. Prabhugoud I. Basarkod, Professor, School of Electronics and Communication Engineering, REVA University. He has 32 years of teaching experience with many research publications in reputed national/international journals and conferences. Some of the works of his research are published in various international journals such as International Journal of Computer Science, Journal of Electronic and Telecommunications, Journal of Telecommunications and Information Technologies, Elsevier International Journal of Computer and Electrical Engineering, and IETE Journal of Education. His research areas of interests include Wireless Networks, Mobile Ad hoc Networks (MANETs), Agent technology, Multimedia communication and wireless sensor networks. Currently he is guiding $6 \mathrm{Ph} . \mathrm{D}$ students. He is a member of Indian society for Technical Education, Institute of Electrical and Electronics Engineers (IEEE), Member Institution of Electronics and Member of Institution of Electronics and Telecommunication Engineers (IETE). 